\title{
Review of oncological findings and complications after elective neck dissection during total laryngectomy: a case series
}

\author{
Mahmoud Elsayed ${ }^{1,2^{*}} \mathbb{D}$, Nada Dwiddar ${ }^{1}$, Amr Allam ${ }^{1}$, Kishan Ubayasiri ${ }^{1}$, Anthony Simons ${ }^{1}$ and Neeraj Sethi ${ }^{1}$
}

\begin{abstract}
Background: This study aims to report the rate of occult lymph node metastasis during elective neck dissection in clinically and radiologically negative neck (cNO) for patients offered total laryngectomy as either primary or salvage treatment. We also compare the rate of complications and recurrence after elective neck dissection during total laryngectomy.

A retrospective review of patients treated in a single teaching hospital in the UK between 2004 and 2020 was performed. We collected the following patient information: age, sex, TNM staging, tumour location and laterality, primary treatment received if recurrent, preoperative imaging, operative details of the elective neck dissection, pathology results, postoperative complications, and recurrence. Inclusion criteria are as follows: squamous cell carcinoma and cNO neck. Exclusion criteria are as follows: second primary tumour in the head and neck, a total laryngectomy because of a dysfunctional larynx, or a previously performed neck dissection.
\end{abstract}

Results: Of the 105 included patients, 66 (63\%) had primary surgery and 39 (37\%) were offered salvage surgery. All cases in the primary group were offered elective neck dissection. Thirty-six patients of the salvage group were offered elective neck dissection. The rate of occult lymph node metastasis was 35\% (23/66) and 5.6\% (2/36) in the primary and salvage groups, respectively. There was no difference in the overall complication rate in both groups, although the specific incidence of pharyngocutaneous fistula in the salvage group was higher compared with the primary group, $14 \%$ vs $7.5 \%$.

Conclusions: We conclude that elective neck dissection is indicated during primary total laryngectomy due to the high rate of occult lymph node metastasis, 35\% (23/66) in our series. Conversely, from our data in the salvage setting, the rate of occult lymph node was very low at $5.6 \%(2 / 36)$. The impact on survival is not known. The decision over elective neck dissection may be made more judiciously on an individual basis in the salvage setting.

Keywords: Laryngectomy (salvage/primary), Head and neck cancer, Squamous cell carcinoma, Neck dissection, Occult lymph node metastasis

\section{Background}

The management of the clinically and radiologically negative neck (cN0) in cases of head and neck cancer has long been a matter of debate. Weiss et al's decision analysis

\footnotetext{
*Correspondence: Mahmoud.elsayed@nhs.net

${ }^{2}$ EENT Building-ENT Department, Queens Medical Centre, Derby Road, Nottingham NG7 2UH, UK

Full list of author information is available at the end of the article
}

has a profound influence for the last three decades and suggested an occult nodal metastasis risk threshold for treatment of $20 \%$, which has become widely accepted [1].

Laryngectomy encompasses treatment for locally advanced malignancies of the hypopharynx and larynx. When undertaking primary laryngectomy for this disease with $\mathrm{cN} 0$, UK guidelines and the National 
Comprehensive Cancer Network (NCCN) guidelines recommend elective neck dissection (END) of levels II-IV $[2,3]$.

In the salvage setting, performing END with laryngectomy is a matter of extensive controversy. Retrospective studies have argued against END based on the rarity of occult neck metastasis, the potential lack of impact on distant metastases, and the morbidity of salvage neck dissections in the cN0 neck [4-8]. However, other studies show an enhanced survival after an END for advanced local disease in the cN0 neck [9]. Others advise an END only in advanced recurrent stage T3-T4, supraglottic, and hypopharyngeal cancer due to the higher rate of occult metastasis [10-12].

A review of the literature shows that the incidence of occult cervical lymph node metastasis in recurrent laryngeal malignancies ranges from 0 to $28 \%$ [4, 5, 7-15]. Hilly et al. reviewed 41 patients undergoing salvage surgery for laryngeal/hypopharyngeal carcinoma. They found a high rate of postoperative complications in those undergoing bilateral END at the time of salvage (pharyngo)laryngectomy), but the differences in regional cancer control and cause-specific survival were not statistically significant [6]. Given the controversy in the literature, this study aims to add further evidence as to whether an END should be performed during salvage laryngectomy by reporting the rate of occult lymph node (OLN) metastasis in our case series. We also compare the rate of complications and recurrence after END in primary and salvage laryngectomies.

\section{Methods}

A retrospective study of patients who underwent laryngectomy either for primary or recurrent cancer of the larynx or hypopharynx in the period June 2004 to December 2020 was undertaken. Relevant cases were identified through our electronic medical record system. Inclusion criteria were primary squamous cell carcinoma (SCC) of the larynx or hypopharynx with cNO at the time of diagnosis. A neck was considered $\mathrm{cNO}$ if no pathological lymph nodes were found during imaging (CT, MRI, PET, and/or ultrasound) and/or physical examination, or if the detected nodes were cytologically benign. We only included patients treated with curative intent. For the included salvage surgery cases, the primary tumour must have been treated with radiotherapy or chemoradiotherapy only. Patients were excluded in cases of laryngectomy for non-functional larynx following radiotherapy or when a neck dissection had previously been performed. The decision to perform surgery with or without neck dissection was taken after discussion in the head and neck cancer multidisciplinary team meeting (MDT). We collected the following patients' information: age, sex, TNM staging, tumour location and laterality, primary treatment received if recurrent, preoperative imaging, operative details of the END, pathology results, postoperative complications, and recurrence. Primary and recurrent laryngeal tumours were classified by the TNM Classification of Malignant Tumours [8th edition, 2016]. In our centre, elective neck dissection was performed in almost all patients who were offered laryngectomy whether primary or salvage. One hundred and five patients who met the inclusion criteria were included in the study. Sixty-six were primary cancers and 39 were recurrent. Patients' characteristics are summarized in Table 1.

All patients were followed up to January 2021, or until the date of death. Recurrences after salvage laryngectomy were confirmed after physical examination, imaging, and biopsy.

Statistical analyses were performed using IBM SPSS Statistics 27. A statistical significance level of 5\% was used. Descriptive statistics were compared using the Pearson chi-square test in case of categorical variables.

Table 1 Summary of patients' characteristics included in the study

\begin{tabular}{|c|c|c|}
\hline Characteristics & $\begin{array}{l}\text { Primary surgery } \\
66 \text { patients }(63 \%)\end{array}$ & $\begin{array}{l}\text { Salvage surgery } \\
39 \text { patients ( } 37 \%)\end{array}$ \\
\hline \multicolumn{3}{|l|}{ Sex } \\
\hline Male & $48(73 \%)$ & $29(74 \%)$ \\
\hline Female & $18(27 \%)$ & $10(26 \%)$ \\
\hline Age: mean & 66.2 years & 69.2 \\
\hline \multicolumn{3}{|l|}{ Tumour subsite } \\
\hline Glottis & $22(33 \%)$ & $25(64 \%)$ \\
\hline Supraglottic & $24(37 \%)$ & $7(18 \%)$ \\
\hline Hypopharynx & $20(30 \%)$ & $7(18 \%)$ \\
\hline \multicolumn{3}{|l|}{ Initial T stage } \\
\hline $\mathrm{T} 1-\mathrm{T} 2$ & $1(1.5 \%)$ & $14(36 \%)$ \\
\hline $\mathrm{T} 3-\mathrm{T} 4$ & 65 (98.5\%) & $25(64 \%)$ \\
\hline \multicolumn{3}{|l|}{ Initial treatment } \\
\hline Radiotherapy & 0 & $32(82 \%)$ \\
\hline Chemoradiotherapy & 0 & $7(18 \%)$ \\
\hline \multicolumn{3}{|l|}{ Imaging performed } \\
\hline CT neck & $62(94 \%)$ & $38(97 \%)$ \\
\hline PET & $14(21 \%)$ & $19(49 \%)$ \\
\hline MRI & $3(4.5 \%)$ & $4(10 \%)$ \\
\hline US & $4(6 \%)$ & $3(8 \%)$ \\
\hline \multicolumn{3}{|l|}{ Neck dissection } \\
\hline Unilateral & $4(6 \%)$ & 0 \\
\hline Bilateral & $62(94 \%)$ & $36(92 \%)$ \\
\hline Not done & 0 & $3(8 \%)$ \\
\hline OLN & 23/66 (35\%) & $2 / 36(5.6 \%)$ \\
\hline Complications & 19/66 (29\%) & $10 / 36(28 \%)$ \\
\hline Recurrence after surgery & $8 / 66(12 \%)$ & $7 / 36(19 \%)$ \\
\hline
\end{tabular}




\section{Results}

\section{Patients}

Sixty-six patients treated with primary laryngectomy were included. Seventy-three percent of patients were males. The average patient age at the time of surgery was 66.2 (range 26-91) years. Of the 66 patients reviewed, the primary tumour subsite distribution was as follows: 22 patients (33\%) glottic carcinoma, 24 patients (37\%) supraglottic carcinoma, and 20 patients (30\%) hypopharyngeal carcinoma (all piriform sinus). One patient was staged as T1-2 (1.5\%), and 65 were staged as T3-4 (98.5\%). Imaging performed before primary laryngectomy included CT of the neck (94\%) and PET (21\%). All patients had either CT or PET scan or both.

For the salvage laryngectomy group, we included thirtynine patients. Seventy-four percent of patients were males. The mean patient age at the time of surgery was 69.2 (range 31-85) years. Of the 39 patients reviewed, the primary tumour subsite distribution was as follows: 25 patients with glottic carcinoma (64\%), 7 patients with supraglottic carcinoma (18\%), and 7 patients with hypopharyngeal carcinoma (18\%) (all piriform sinus). Fourteen patients were staged as T1-2 (36\%), and 25 were staged as T3-4 (64\%). Imaging performed before salvage laryngectomy included CT of the neck (97\%) and PET (49\%). All patients had either CT or PET scan or both. Since June 2016, the date we started requesting PET scans in our hospital, 21 out of 25 patients had PET scan (84\%). The 4 salvage cases who did not have PET scans had recurrent glottic cancer.
Neck dissection outcome (Fig. 1)

An END was performed on all of them at the time of the primary laryngectomy patients. Of the performed ENDs, $62(94 \%)$ were bilateral and $4(6 \%)$ unilateral. Twentythree patients had OLN metastasis (35\%). OLN metastasis occurred in 7 patients with glottic cancer (30\%), 10 patients with supraglottic cancer (44\%), and 6 patients with hypopharyngeal cancer (26\%). Occult lymph nodes were unilateral in 17 patients (74\%) and bilateral in 6 patients (26\%). Lateralised tumours had either ipsilateral or bilateral nodal disease; none had isolated contralateral nodal disease. See Table 2.

For the salvage laryngectomy, END was performed on 36 out of 39 (92\%). Of the performed ENDs, all patients received bilateral neck dissection. Only 2/36 patients had OLN metastasis (5.6\%). Both patients had recurrent glottic cancer; one case was T2 and the other was T4a, and both developed a recurrence in the first year of their follow-up.

\section{Complications}

As for the primary laryngectomy patients, 29\% (19/66) of patients developed a postoperative complication. Quite similarly, $28 \%(10 / 36)$ of the salvage patients who had END developed a postoperative complication. Antrolateral thigh flap is used routinely for reconstruction after salvage laryngectomy in our hospital. One patient out of the three who underwent salvage laryngectomy without END developed a complication. Complications included

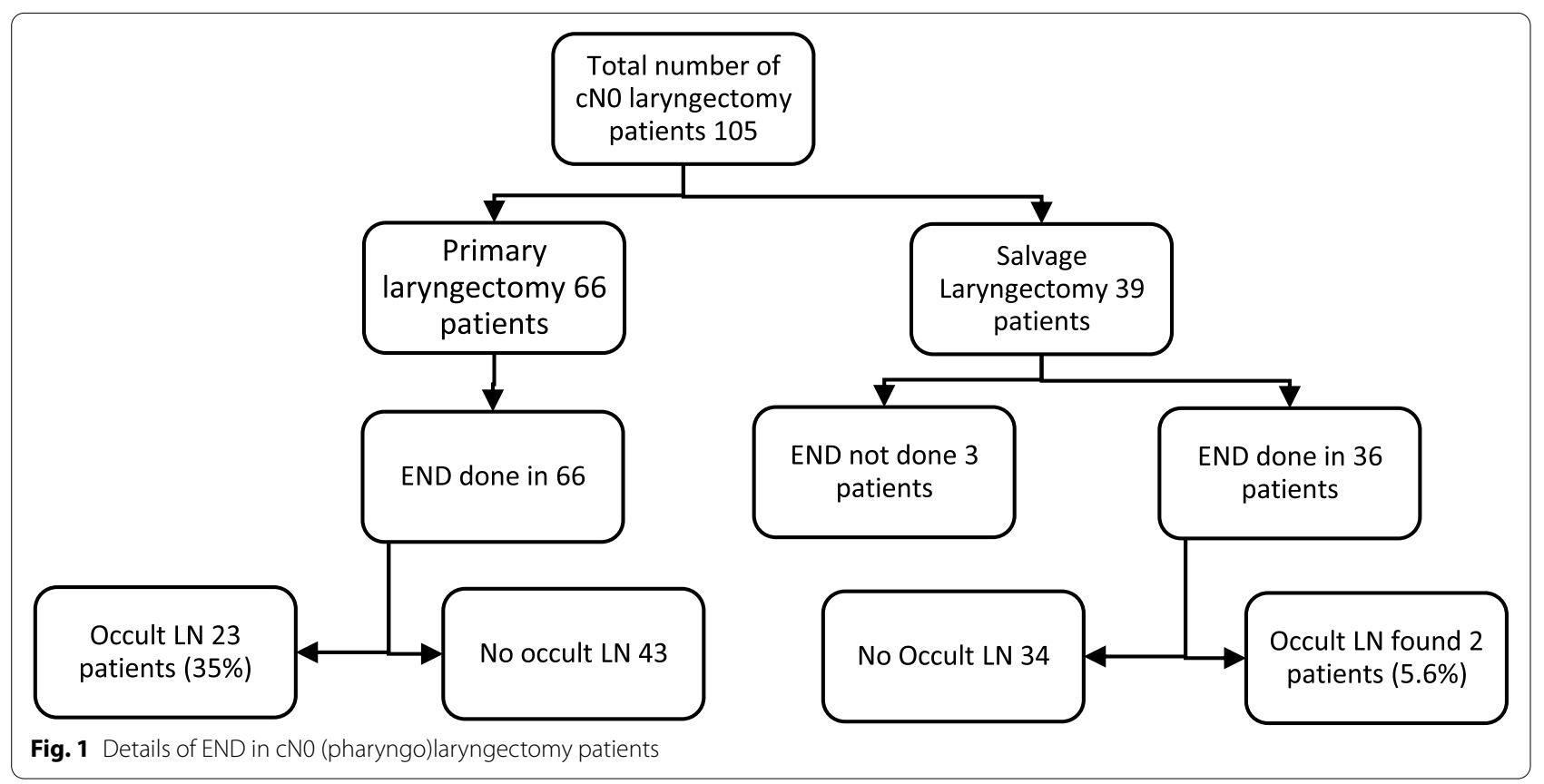


Table 2 Distribution of occult lymph node metastases in primary (pharyngo)laryngectomy

\begin{tabular}{llll}
\hline & Glottic (22 patients) & Supraglottic (24 patients) & $\begin{array}{l}\text { Hypopharynx } \\
\text { (20 patients) }\end{array}$ \\
\hline Total OLN (23 patients) & $7 / 23(30 \%)$ & $10 / 23(44 \%)$ & $6 / 23(26 \%)$ \\
Ipsilateral OLN (17 patients) & 4 & 7 & 6 \\
Bilateral OLN (6 patients) & 3 & 3 & 0 \\
\hline
\end{tabular}

Table 3 Complications after primary and salvage (pharyngo) laryngectomy

\begin{tabular}{llll}
\hline & Primary & Salvage & p value \\
\hline Total & $19 / 66(29 \%)$ & $10 / 36(28 \%)$ & 0.914 \\
Pharyngocutaneous fistula & $5(7.5 \%)$ & $5(14 \%)$ & 0.306 \\
Neck collection (infection) & 7 & 3 & 0.712 \\
Bleeding & 1 & 1 & 0.66 \\
Wound breakdown & 6 & 1 & 0.223 \\
\hline
\end{tabular}

bleeding, neck collection (infection), and pharyngocutaneous fistula. See Table 3.

\section{Oncological outcome (recurrence)}

Eight patients had recurrent cancer (12\%) after primary laryngectomy with END. Out of those, seven patients $(88 \%)$ had OLN metastasis on histopathology, and one had no OLN. Six patients suffered recurrence in the first 5 years. As for the salvage group, seven patients $(7 / 36)$ (19\%) suffered recurrent cancer after surgery. All these cases that developed recurrence had laryngectomy with END. All recurrences happened in the first 3 years.

\section{Discussion}

This study reports the rate of OLN metastasis in cases offered END during laryngectomy either primary or salvage, in a single teaching hospital in the UK. We also examined the rate of complications and recurrence after END in both groups. END is recommended for primary treatment of advanced laryngeal cancer [2, 3]. Neck dissection is suggested if the risk of nodal disease is greater than $15 \%$ to $20 \%[16,17]$. In our cohort, the overall OLN rate was $35 \%$, suggesting a benefit for elective neck dissection in primary laryngectomy patients. OLN metastasis occurred on the ipsilateral side of the cancer in $74 \%$. Bilateral OLN metastasis occurred in only 6 cases (26\%); three of these were supraglottic cancer, and the other three cases were glottic cancer involving the anterior commissure. In cases of lateralised hypopharyngeal cancer, OLN metastases were only found in the ipsilateral neck.
For salvage laryngectomy, the decision to perform an END could be based on the risk of OLN. In our study, only 2 patients had an occult metastasis (5.6\%), which is low compared to the reported rate in the literature $(0-28 \%)$ [4, 5, 7-15]. The wide spread in variance reported for OLN metastasis in the literature can be explained by differences in study populations, e.g., patient characteristics, number of included patients, preoperative radiological imaging, and treatment modalities. The 2 patients with occult metastasis in our salvage cohort initially presented with a T4NOM0 glottic tumour. Post-operative pathological examination showed laryngeal cartilage invasion and perineural involvement. Previous research showed a significantly higher risk of occult metastasis in cases of cartilage invasion and perineural invasion [10].

In our study, the complication rate was similar between the primary and salvage laryngectomy groups $(p=0.914)$. Our overall complication rate of $29 \%$ for primary laryngectomy is comparable to rates that have been reported in other recent series and systematic reviews [18-20]. A total complication rate of $28 \%$ for the salvage laryngectomy group is reported, but this is still below the range found in the literature (43-66\%) $[5,7,15]$. Higher complication rates have been reported by some studies with salvage laryngectomy where a (unilateral or bilateral) END was performed [4, 5, 7]. The incidence of pharyngocutaneous fistula in the salvage setting was higher compared to the primary group ( $p=0.306), 14 \%$ vs $7.5 \%$ which is consistent with the literature. See Table 3 [21].

Whether to perform END during salvage laryngectomy and whether this should be bilateral or unilateral remains controversial, due to a lack of evidence. In our cohort, 36 END were performed, all of which were bilateral. In total, 7 out of the 36 patients who had END during salvage laryngectomy developed a recurrence. Five of these were lung metastases (one of which had synchronous bone metastasis to the humerus) and 2 developed local neck recurrences. Although low rates of OLN may seem suggestive of a lack of necessity to undertake END, the lack of impact of disease specific or overall survival is a vital issue. END has been demonstrated to be of survival benefit in other subsites of head and neck cancer (e.g., 
oral cavity) despite previous reports suggesting low rates of OLN [22].

\section{Conclusion}

We conclude that END is indicated in cases with primary advanced laryngeal and hypopharyngeal cancer due to the high rate of OLN metastasis, $35 \%(23 / 66)$ in our series. Conversely, from our data in the salvage setting, the rate of OLN was extremely low at 5.6\% (2/36). It is difficult to draw definitive conclusions regarding the application of END in the salvage setting as data regarding the impact on survival is lacking. However, neck dissection can certainly be applied judiciously to each individual patient, weighing the risks and benefits for each, rather than a simple blanket policy for all. Although this is a small retrospective series, the greatest value of this study is to add to the literature the finding that END in the salvage setting is low yield and may not worth pursuing.

We did not find any difference in the overall complication rate between primary and salvage surgery ( $p=0.914$ ), 29\% and 28\%, respectively. However, the specific rate of pharyngocutaneous fistula was higher in the salvage setting compared to primary surgery.

\section{Limitation}

The main limitations of our study were its retrospective nature and the limited number of cases available for analysis. It was difficult to draw any robust conclusion on survival between the patients who underwent salvage laryngectomy with END and those without END, due to the limited number of cases who had undergone (pharyngo) laryngectomy without END, 3/39 (8\%).

\footnotetext{
Acknowledgements

The abstract of this study was presented as a poster in The Joint European Congress on Head and Neck Oncology (ECHNO) and the International Congress on Innovative Approaches in Head and Neck Oncology (ICHNO) July 2021.
}

\section{Authors' contributions}

Authors are responsible for correctness of the statements provided in the manuscript. All authors contributed to the study conception and design. Material preparation, data collection and analysis were performed by ME, AA and ND. The first draft of the manuscript was written by ME. AS, KU and NS commented on all versions of the manuscript. All authors read and approved the final manuscript.

\section{Funding}

None to declare.

\section{Availability of data and materials}

Yes, all data generated or analysed during this study are included in this published article.

\section{Declarations}

Ethics approval and consent to participate

The study was registered with the local research and development Department in Nottingham University Hospitals (Reference number 21-00c - Date: 4th of May 2021). Ethical approval was waived due to the retrospective nature of the study. All patients' details were anonymised before data analysis. Access was granted to the patients' data after approval from the Audit - information Governance department approval. Consent to participate is not applicable due to the retrospective nature of the study.

\section{Consent for publication}

No individual patient data, not applicable.

\section{Competing interests}

The authors declare that they have no competing interests.

\section{Author details}

${ }^{1}$ Department of Otolaryngology Head and Neck Surgery, Nottingham University Hospitals, Nottingham, UK. ${ }^{2}$ EENT Building-ENT Department, Queens Medical Centre, Derby Road, Nottingham NG7 2UH, UK.

Received: 10 September 2021 Accepted: 11 October 2021

Published online: 03 November 2021

\section{References}

1. Weiss MH, Harrison LB, Isaacs RS (1994) Use of decision analysis in planning a management strategy for the stage NO neck. Arch Otolaryngol Head Neck Surg. 120(7):699-670

2. National Comprehensive Cancer Network. Head and Neck Cancers (Version 1.2020) To view, visit https://jnccn.org

3. Jones TM, De M, Foran B, Harrington K, Mortimore S (2016) Laryngeal cancer: United Kingdom national multidisciplinary guidelines. J Laryngol Otol. 130(S2):S75-S82

4. Deganello A, Meccariello G, Bini B, Paiar F, Santoro R, Mannelli G, Gallo O (2014) Is elective neck dissection necessary in cases of laryngeal recurrence after previous radiotherapy for early glottic cancer? J Laryngol Otol 128(12):1089

5. Basheeth N, O'Leary G, Sheahan P (2013) Elective neck dissection for NO neck during salvage total laryngectomy: findings, complications, and oncological outcome. JAMA Otolaryngol Head Neck Surg 139(8):790-796

6. Hilly O, Stern S, Horowitz E, Leshno M, Feinmesser R (2013) Is there a role for elective neck dissection with salvage laryngectomy? A decisionanalysis model. Laryngoscope 123(11):2706-2711

7. Bohannon IA, Desmond RA, Clemons L, Magnuson JS, Carroll WR, Rosenthal EL (2010) Management of the NO neck in recurrent laryngeal squamous cell carcinoma. Laryngoscope 120(1):58-61

8. Pezier TF, Nixon IJ, Scotton W, Joshi A, Guerrero-Urbano T, Oakley R, Jeannon JP, Simo R (2014) Should elective neck dissection be routinely performed in patients undergoing salvage total laryngectomy? J Laryngol Otol 128(3):279

9. Hilly O, Gil Z, Goldhaber D, Amit M, Biadsee A, Popovtzer A, Shvero J, Cohen J, Fliss D, Feinmesser R, Bachar G (2015) Elective neck dissection during salvage total laryngectomy-a beneficial prognostic effect in locally advanced recurrent tumours. Clin Otolaryngol 40(1):9-15

10. Yao M, Roebuck JC, Holsinger FC, Myers JN (2005) Elective neck dissection during salvage laryngectomy. Am J Otolaryngol 26(6):388-392

11. Wax MK, Touma BJ (1999) Management of the NO neck during salvage laryngectomy. Laryngoscope. 109(1):4-7

12. Koss SL, Russell MD, Leem TH, Schiff BA, Smith RV (2014) Occult nodal disease in patients with failed laryngeal preservation undergoing surgical salvage. Laryngoscope. 124(2):421-428

13. Farrag TY, Lin FR, Cummings CW, Koch WM, Flint PW, Califano JA, Broussard J, Bajaj G, Tufano RP (2006) Neck management in patients undergoing postradiotherapy salvage laryngeal surgery for recurrent/persistent laryngeal cancer. Laryngoscope. 116(10):1864-1866

14. Amit M, Hilly O, Leider-Trejo L, Popovtzer A, Gutfeld O, Shvero J, Fliss DM, Cohen JT, Bachar G, Gil Z (2013) The role of elective neck dissection in patients undergoing salvage laryngectomy. Head Neck 35(10):1392-1396

15. Freiser ME, Ojo RB, Lo K, Saint-Victor S, Bollig C, Nayak CS, Sargi ZB (2016) Complications and oncologic outcomes following elective neck dissection with salvage laryngectomy for the N0 neck. Am J Otolaryngol 37(3):186-194 
16. Robbins KT (2000) Indications for selective neck dissection: when, how, and why. Oncology (Williston Park, NY) 14(10):1455-1464

17. Martin H (1951) The case for prophylactic neck dissection. Cancer. 4(1):92-97

18. Goepfert RP, Hutcheson KA, Lewin JS, Desai NG, Zafereo ME, Hessel AC, Lewis CM, Weber RS, Gross ND (2017) Complications, hospital length of stay, and readmission after total laryngectomy. Cancer. 123(10):1760-1767

19. Hasan Z, Dwivedi RC, Gunaratne DA, Virk SA, Palme CE, Riffat F (2017) Systematic review and meta-analysis of the complications of salvage total laryngectomy. Eur J Surg Oncol (EJSO) 43(1):42-51

20. Sullivan CB, Ostedgaard KL, Al-Qurayshi Z, Pagedar NA, Sperry SM (2020) Primary laryngectomy versus salvage laryngectomy: a comparison of outcomes in the chemoradiation era. Laryngoscope. 130(9):2179-2185
21. Paleri V, Drinnan M, van der Brekel MWM, Hinni ML, Bradley PJ, Wolf GT et al (2014) Vascularized tissue to reduce fistula following salvage total laryngectomy: a systematic review. Laryngoscope. 124(8):1848-1853

22. Abu-Ghanem S, Yehuda M, Carmel NN, Leshno M, Abergel A, Gutfeld O, Fliss DM (2016) Elective neck dissection vs observation in early-stage squamous cell carcinoma of the oral tongue with no clinically apparent lymph node metastasis in the neck: a systematic review and meta-analysis. JAMA Otolaryngol Head Neck Surg 142(9):857-865

\section{Publisher's Note}

Springer Nature remains neutral with regard to jurisdictional claims in published maps and institutional affiliations.

\section{Submit your manuscript to a SpringerOpen ${ }^{\circ}$ journal and benefit from:}

- Convenient online submission

- Rigorous peer review

- Open access: articles freely available online

- High visibility within the field

Retaining the copyright to your article

Submit your next manuscript at $\boldsymbol{\nabla}$ springeropen.com 